\title{
Health surveillance, biosafety and emergence and re-emergence of infectious diseases in Brazil
}

\section{Authors}

Telma Abdalla de Oliveira Cardoso

Marli BM de Albuquerque Navarro

Cristina Costa $\mathrm{Neto}^{2}$ Josino Costa Moreira ${ }^{3}$

${ }^{1}$ Researcher of Biosafety Office. National School of Public Health. Fundação Oswaldo Cruz, Rio de Janeiro, Brazil.

${ }^{2}$ Researcher of Biologic Science Department. National School of Public Health. Fundação Oswaldo Cruz, Rio de Janeiro, Brazil. ${ }^{3}$ Researher at the Center for Worker's Health and Human' Ecology Studies. National School of Public Health. Oswaldo Cruz Foundation - Researher at the Center for Worker's Health and Human' Ecology Studies. National School of Public Health. Oswaldo Cruz Foundation.

Submitted on: 08/20/2009 Approved on: 10/30/2009

Correspondence to: Telma Abdalla de Oliveira Cardoso

Av. Brasil 4036, sala 716, Manguinhos, Rio de Janeiro, RJ, Brazi CEP: 21040-361

Phone/Fax: +55-2125905988

E-mail:

abdalla@fiocruz.br

We declare no conflict of interest.

\begin{abstract}
The present paper presents compulsory notification data for infectious diseases and epidemiologic ones recorded at the Center for Strategic Information and Health Surveillance (CIEVS) for the period of March 2006 to April 2007. Data is presented in accordance with geographic distribution, time and risk classification of the etiologic agents found, according to Ministry of Health regulations. The importance of this epidemiologic surveillance system is presented, debating the main topics required for quality improvement and information analysis. It is concluded, from the analysis of epidemiologic events and their relation to risk management, that the compulsory notification system in Brazil is incomplete, irregular, delayed and, in a large percentage of cases, notification cannot be completed and the agent may not be identified. Quality of data varies from one region to another and from county to county within the same region. There is a high proportion of cases in which the etiologic agent is unknown and, in such cases, a high lethality is expected, establishing a high risk exposure condition for those health professionals involved in health surveillance. From these data, the study points out the need to improve the surveillance system and strengthens the idea of building maximum containment laboratories.
\end{abstract}

Keywords: biosafety, epidemiologic surveillance, infectious diseases, public health.

[Braz J Infect Dis 2010;14(5):526-535]@Elsevier Editora Ltda.

\section{INTRODUCTION}

Biosafety appears in a globalized world as an increasingly necessary field of knowledge to respond to the complexity presented particularly by biological risk, from the reality of emerging and re-emerging infectious diseases, bioterrorism, environmental predation, and commitment to ecological heritage of the planet and its equilibrium. These aspects are associated with the circulation process of people and goods, migratory flows, poverty, wars, transportation, among others, depicting a context which places important and complex challenges to public health and, especially, to epidemiology.

The risk associated with new etiological agents of high lethality, which may turn into pandemic events, either known and/or poorly known, is a growing concern. ${ }^{1}$ Analyses on the theme state that there is a latent risk linked to the circulation of viruses, which can, within a few hours, reach continents simultaneously, through air trips, thereby dispersing lethal biological agents and demanding effective epidemiological surveillance systems from governments, by setting up laboratory networks and notification systems as part of an effective biosafety infrastructure..$^{2-5}$ The challenges placed on public health have been discussed, notwithstanding that in terms of global concerns; they entail factors configuring the construction of worldwide calamity implementation. ${ }^{6}$ Among these challenges, implementation of Biosafety has also been placed as a field which encompasses the complexity of risk monitoring and its articulation with health surveillance.

Countries have been assigning priority to preventive and control actions in order to avoid or minimize the effects of infectious diseases, through surveillance-geared policies, on epidemiological, laboratory and clinical basis. ${ }^{7}$

The efficiency of epidemiological surveillance programs requires the setting of an infrastructure essential for information systems, enabling them to establish a strategy based on a warning and rapid response mechanism: the setting up of a laboratory network, organized in a hierarchical manner, appropriately equipped, with qualified human resources, able to ensure the necessary biosafety 
conditions. ${ }^{8,9}$ Investments in these areas nowadays present theirselves as imperatives for the countries as in the effectiveness and efficiency of risk control, especially biological one, depending on prevention policies, with continuous awareness of the changes in the habitual patterns of diseases and in the emergence of others. ${ }^{10}$

\section{Brazilian endeavors on biosafety}

In order to develop a surveillance strategy based on clinical and laboratory epidemiologic data, in 1995, the Scientific and Technologic Development Coordination of the Brazilian Ministry of Health conducted a workshop aimed at discussing the Brazilian Scientific and Technologic Capacity Building Project for Emerging and Re-Emerging Infectious Diseases, where the common thought was to increase human resources abilities of Brazilian institutions in the field, since biosafety would be a priority issue in any process, whether with regard to facilities improvement or to change of behavior based on new information. ${ }^{11}$

The main challenge turned out to be how to work these issues out. It was necessary to know the real conditions of the country's institutions when it comes to quality in Biosafety. To this end, a Biosafety Office (NUBio) of Oswaldo Cruz Foundation (FIOCRUZ), which had already been carrying out a survey on the risks, was contacted, and in 1996, a Scientific and Technological Capacity Building Program in the Biosafety Field was implemented.

This Program had two basic axes of action: risk identification and mapping, and capacity building of human resources focusing on the main gaps identified on the survey. The Public Health Laboratories (LACEN) from the states of São Paulo, Minas Gerais, Pernambuco, Pará and Bahia took an active role in the study, as well as FIOCRUZ regional research centers. The main results of this survey were identified as awareness of the issue of some 900 health workers who participate in courses and creating biosafety internal committees in the institutions involved. ${ }^{12}$

In the year 2000, the Brazilian Public Health Laboratories Coordination (CGLAB), today part of the Health Surveillance Secretariat (SVS), has, in cooperation with the Centers for Disease Control/EUA and Nubio/FIOCRUZ, set up a capacity building program aimed at training multiplier subjects in biosafety. According to this group's view, biosafety issues go far beyond genetic manipulation and its ethical issues, limited to a small sample of highly specialized laboratories or facilities. Funding of laboratories belonging to the National Public Health Network was set up as a priority. This net is composed of LACEN, linked to the State's Secretariat of Health (SES) and by reference institutions linked to the MS, such as FIOCRUZ and Pasteur Institute (SP). These labs are responsible for most of disease diagnosis under surveillance and, therefore, use techniques for handling infectious agents that may be considered routinely risky.
Within the three strategies, the following main areas of action were generally defined: continued education for college and technical level personnel; setting up of basic requirements for the minimum facility and equipments conditions; good Laboratory Practices in handling pathogenic biologic agents, transport, transfer and storage of samples, among other rules; implementing internal biosafety committees; compliance, reform or building of laboratory areas suited for Biosafety Level 3 (BSL3).

From 2000 on, 273 multipliers were capacitated, representing all the 27 Brazilian LACEN.

Capacity building of college graduates on biosafety, aiming at the spread of different reference centers of public health in Brazil, has resulted in a management and surveillance policy. Making relevant information available and working out control and prevention risk strategies has started to be devised in Brazil. These graduates, upon returning to their original states, have set up a process of multiplying the information, still active up to now. At present, this program has trained about 4,000 people. ${ }^{12}$

The SVS has been putting into force since 2005 the implementation of a network of BSL3 within the National Public Health Laboratories Network. These lab BSL3 areas are equipped for the development and performance of studies related to diagnosis and identification of risky biologic agents, such as Hantanvirus or the Anthrax bacteria.

These laboratory areas should be considered reference for Latin America, since they shall represent the strengthening of epidemiologic surveillance capacity within the whole region, especially for airborne diseases, caused by risk group three infectious agents.

At present, 13 BSL3 facilities are ready to use, as follows: Instituto Adolfo Lutz/SES/SP.

Laboratório Central de Saúde Pública do Estado do Ceará/SES/CE

Laboratório Central de Saúde Pública do Distrito Fed$\mathrm{eral} / \mathrm{SES} / \mathrm{DF}$

Centro de Referência Hélio Fraga/FIOCRUZ/RJ

Instituto Evandro Chagas/SVS/MS/PA

Instituto Octávio Magalhães/FUNED/SES/MG

Centro de Pesquisa Aggeu Magalhães/FIOCRUZ/PE

Laboratório Central de Saúde Pública do Estado do Rio Grande do Sul/SES/RS

Instituto Pasteur/SES/SP

Departamento de Virologia do IOC/FIOCRUZ/RJ

Instituto de Medicina Tropical/IMTM/SES/AM

Centro de Pesquisa Gonçalo Muniz/FIOCRUZ/BA

Centro de Pesquisa em Medicina Tropical/CEPEM/SES/RO

Training and capacity building of the researchers that will work in the BSL3 areas has also started in 2005. The course is being ministered by professionals from FIOCRUZ and from Universidade de São Paulo. 
Since Brazil is a continent-sized country bordering almost all Latin American countries and bearing several biomes, including those on the borders, still not completely known, it displays social and economic features that may render the country vulnerable to such risks. The lack of a chain of containment laboratories organized in a hierarchical manner and of a maximum containment laboratory, jeopardizes diagnostic activities, as the biological material is forwarded overseas, since investigations involving group 4 biological agents cannot be made internally. This deficiency also impacts the investments in technologies meant for the development of immunobiological and pharmacological products, as well as diagnostic inputs.

This work aims at discussing, through the data acquired from the Center for Strategic Information and Health Surveillance (CIEVS), with the SVS; the epidemiologicallyrelevant, notified and collected events, from March 2006 to April 2007; in order to support the need for setting up a maximum-containment laboratory unit in Brazil, based on the country's particularities in epidemiological terms, its important biodiversity and its social and economic reality.

\section{Center for strategic information and health surveillance}

The CIEVS was founded in 2006, for the follow-up of diseases demanding compulsory reporting. It bears human and technological resources to carry out continuous collection and monitoring of epidemiological emergencies from a warning issued by the State Secretariat of Health (SES) and City Department of Health, by practitioners or by health services all over the country. It provides support, within the health surveillance scope, to the demands linked to the changes in the occurrence patterns of infectious diseases and in the transmission dynamics of its agents, as well as to unusual cases, epidemiological emergency conditions of a biological, chemical or unknown nature, catastrophes and others which comprise outbreaks or epidemics. This information is immediately reported to the SES and evaluated by the SVS, in order to confirm their epidemiological relevance.

The Ministry of Health published in 1999, with the purpose of establishing criteria which would allow measuring the importance of a disease from a national surveillance perspective, a list of compulsory-notification of diseases constantly updated, following incorporation of new strategies and technologies. Thus, suspicious or confirmed cases of the West Nile Fever and of Severe Acute Respiratory Syndrome (SARS) were included in the list, as from 2003, by Ordinance $n^{\circ} 2325$. In 2005, Ordinance $n^{\circ} 33$ inserted the cases of Creutzfeld-Jakob Disease (CJD), syphilis in pregnant women, Acute Febrile Icterohemorrhagic Syndrome, in addition to post-vaccination adverse events. In 2006, Ordinance $n^{\circ} 5$ made notification of suspected or confirmed Human Influenza cases by a new subtype compulsory. ${ }^{13}$
Recently, the increase in number of morbid events, deemed emergent, whose etiology is unknown, led to the definition of "unusual diseases", featured as the occurrence of cases or deaths by a disease of an unknown origin or cases of known diseases which display some alteration to their epidemiological pattern. These cases are reported to the sanitary authorities independently of being found in the National List of Compulsory Notification Illnesses and Diseases. The inclusion of this concept strengthens the strategic dimension of the system in detecting events of an unknown origin or of development of diseases which deviate from an established pattern.

Ordinance no 5 also presents a list of immediate notification illnesses and diseases which, on account of their high dissemination potential, or risks to public health, risk to peoples traffic, high mortality and morbidity rate need follow-up by the Ministry of Health. ${ }^{13}$

\section{METHODS}

Data secured from the CIEVS, from March 2006 to the second week of the month of April 2007 were analyzed. Data organization and analysis followed relevance criteria regarding the events reported and their relationship with the manifest or latent risk potential, pointing out to the discussion on investments in Biosafety with regards to building up a maximum containment (BSL4) laboratory infrastructure and ensuring qualification of health professionals, indicating the importance of the debate on the units location, according to the reporting system.

\section{RESULTS AND DISCUSSION}

Presentation of results and their discussion were carried out in two ways: by the events (diseases, outbreaks, illnesses or emergencies) and by chance (number of persons affected by the event).

\section{Numerical quantitative}

Two hundred and forty-seven events were totalized in the covered period, 180 being in 2006 and 67 in 2007, with an average of four events per week. It was ascertained that June 2006 was the record month in the number of reports.

In event systematization by number and by week and month, it was determined that the weeks: $1^{\text {st }}$ in June, last in September; $1^{\text {st }}$ in November and $3^{\text {rd }}$ in December 2006 were those which displayed the greatest number of events, respectively. The increase in frequency of reports in these weeks corresponds to the rainy season in the Northeast Region, which has set off an increase in flu syndrome, diarrhea and dengue fever cases in several capital cities. Average time between beginning of symptoms and notification was 14 days, with an interval between 0 and 218 days ( $\sim 7$ months). It is important to point out that only $38 \%$ of the events reported display information on the beginning date of symptoms. 
Moreover, no events were reported in the $2^{\text {nd }}, 3^{\text {rd }}$ and $4^{\text {th }}$ weeks of March 2006 and in the $3^{\text {rd }}$ week of February 2007. It is assumed that the lack of reports in the first weeks of March 2006 is related to the CIEVS putting-in-place phase.

\section{Spatial distribution of events}

Figure 1 presents the spatial distribution of events reported per Brazilian state. One observes that the states which bore the greatest number of events were as follows: SP (26), MG (25), BA (25), RJ (22) and PA (21). A greater epidemiological framework is shown in the Southeast Region. Conversely, data in the North Region (AC, RR, RO and AP), where there is the occurrence of diseases of compulsory reporting as arboviruses, displays underreporting. It is also important to point out the low level of event reporting in some Northeast Region states, such as AL and PB, and the total absence of events in SE, thus demonstrating deficiency in the regional epidemiological surveillance system.

In the distribution of events per geographical region, one observes that the region with the greatest number of reports is the Southeast $(30.2 \%)$ - a result of SES operation as well as that of public health institutions of reference located therein. The Northeast Region, despite underreporting or total lack of reporting in some of its states, presents $27.2 \%$, corresponding to the second region with the highest number of notifications. This percentage is due to the large number of reports in BA. The North Region, despite PA, having been the fifth state in the number of reports, accounts for only $16.6 \%$ of the reports. The South Region presents $13.2 \%$ and the Center-West Region $12.8 \%$.

The low representativeness may stem from a trend to notify more serious cases, of those under hospital care and those which are being focused by the media at the expense of those displaying benign features, even though the latter may comprise the main sources of infection.

According to the Centers for Disease Control and Prevention, ${ }^{14}$ underreporting is frequently associated to

Figure 1: Distribution of disease events reported to CIEVS, according to the State, from March 2006 to May 2007.

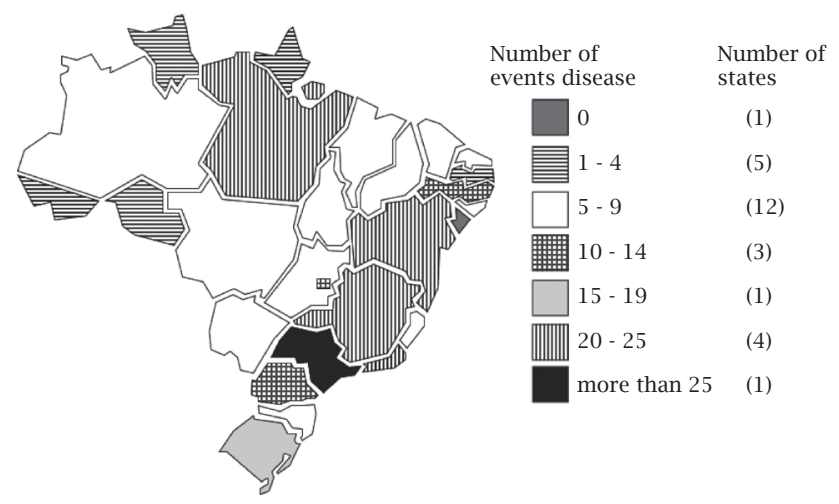

the lack of: knowledge from health professionals about the importance and the necessary reporting procedures; knowledge of the list of diseases contemplated by the surveillance systems; motivation in compliance with reporting procedures, on account of the time consumed in filling out the cards and by the failure to return the analyzed information, with the pertinent technical recommendations; the health professionals' concern referring to breach of data confidentiality, and to the professionals lack of awareness, regarding the public health relevance of the diseases submitted to surveillance.

The participation of health professionals is a critical point in data collection quality; therefore, information given to these teams, highlighting the importance of disease notification toward service enhancement should be a priority in education and training. However, it is important to point out that the adherence of health professionals to systematic notification is, for its most part, conditioned to surveillance response, that is, to the frequency and speed with which it returns, to these professionals, analyzed information, added to technical recommendations applicable to the enhancement of health assistance services.

In Brazil, a way to reach wider participation of notifying professionals would be the inclusion of the Community Health or National Family Health Agent Program in the logistic and practices of surveillance.

Another issue linked to underreporting is the excessive emphasis given to medical assistance upon the initial phase of implementation of the Brazilian National Health Care System, at the expense of the health programs which bear as a feature being based on epidemiological criteria so as to set up priorities.

\section{Types of diseases}

Figure 2 presents the types of diseases reported during the period of research, which displayed the greatest number of events recorded. They were as follows:

Acute Chagas Disease - 22 events. Initially, this quantitative was related to a possible outbreak but it was actually deemed as a result of the improvement in disease surveillance, mainly in the North Region, where one finds cases of oral transmission through the ingestion of "açaí" in PA and AM.

Meningitis - 19 events, occurring mainly in 2006. The states of SP (6), MG (2) and PR (2) displayed the greatest number of cases.

Unknown-etiology syndromes - 18 events. The greatest number of events occurred in 2006 (15 events). During the period reviewed, the events displayed greater distribution in the Southeast (7), Northeast (6) and North (3). 
Figure 2: Number of disease events reported to CIEVS, in the country, from March 2006 to April 2007.

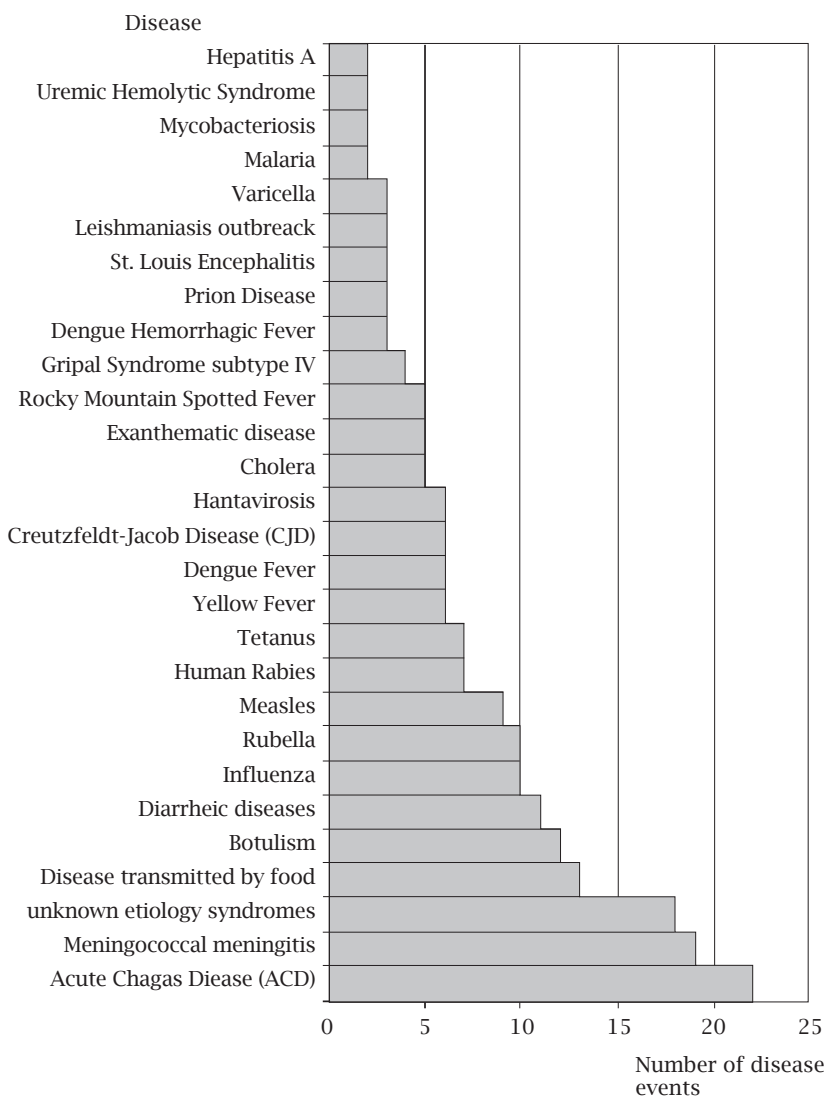

A change to the outlook is ascertained upon the grouping of events by great categories of pathologies, in which exanthematic diseases (lumping together Measles, Rubella and Varicella) have become the group with the greatest number of events in the period reviewed (27). This is due to the fact of the Rubella outbreaks in the States of MG and RJ and of Measles in BA. The arboviruses (lumping together Dengue fever, Hemorrhagic Dengue Fever, Yellow Fever, Wild-Type Yellow Fever, Saint Louis encephalitis, hemorrhagic fevers, and Oropouche Fever) start to display a significant number of occurrences (20). Finally, respiratory syndromes (lumping together Influenza events, SARS, respiratory syndromes, specific cases of suspected H5N1 and epizooties (death of birds) related to these) also display a representative number (16). It is important to highlight that prion diseases (diseases caused by prions and CJD) presented nine occurrences.

\section{Risk of etiological agent involved in the event reported}

Brazil classifies the etiological agents according to their risk in order to relate them to Biosafety recommendations. This classification groups up the biological agents in four risk classes, growing in the degree of containment and complexity in the level of protection. ${ }^{15}$ It is an important instrument to support risk assessment, which shall determine practices and procedures for the handling of infections agents and materials, safety equipment and engineering and architectural aspects of the environments. It is important to point out that any information about the handled biological agent which may lead to alterations in virulence patterns, pathogenicity, antibiotic resistance or other factors, shall imply in stricter containment criteria.

Distinction between risk groups of pathogens involved in the events reported was performed by means of the national biological agent risk classification ${ }^{15}$ thus leading to Figure 3. The diseases which did not have their etiological agent diagnosed are named as "unknown".

One observes the predominance of diseases whose etiological agent belongs to risk group 2, thus ascertaining that most etiological agents handled in the Country fall in this group (72\% of the events), such as Rubella, Measles, Dengue Fever, Meningococcal Meningitis, Chagas disease and Influenza viruses, which have caused the highest number of occurrences.

The etiological agents classified as group 3 are represented along the period reviewed with a steady frequency and average, and account for 19\% of the events reported. This group comprises Hantaviruses, prions (registered as prionic diseases and CJD), the cases of wild-type Yellow Fever and Botulism. Health professionals handling materials suspected of containing agents of this risk group should be mandatorily protected as to the risk of airborne transmission. They have to use, among personal protection equipment, respiratory protection masks with greater filtering effectiveness (N95- filtering effectiveness of $95 \%$ of the particles up to $0.3 \mu$ ). Considering the risk to professionals, public health and to the environment, the amount of events whose etiological agents are unknown is significant ( $9 \%$ of the events reported). These are also of frequent occurrence throughout the period reviewed. These events appears as an important indicator for the professionals as upon absence of specific information on the etiological agent of the outbreak, the professionals have to be ready to perform risk assessment correctly, by using the biological agent risk classification as a

Figure 3: Disease Events reported to CIEVS, in the country, from March 2006 to May 2007, shown by month and year, according to the etiologic agent's risk classification group or diagnosed disease.

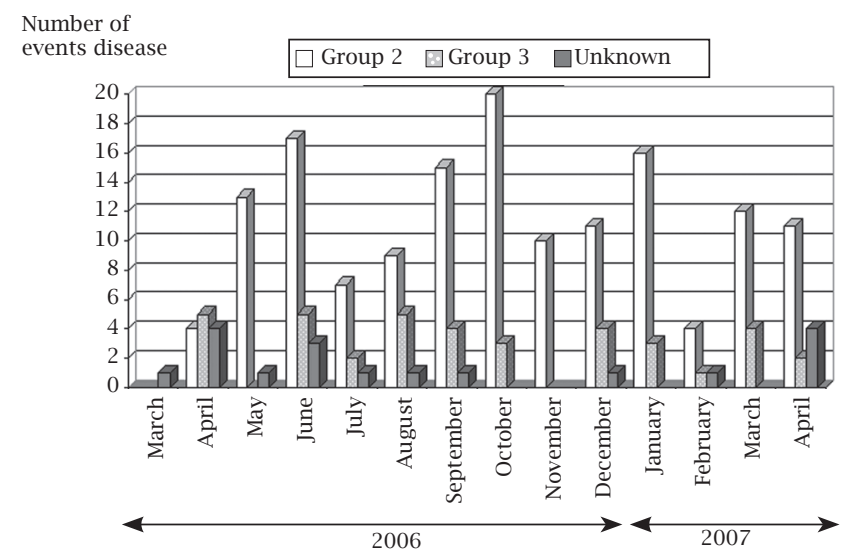


guideline in order to establish appropriate preventive and containment measures to be carried out. These events should be worked on within maximum containment conditions, as they are pathogens which do not bear information on their forms of transmission, thereby increasing the risk. This demonstrates the importance of qualification of the professionals involved, regarding the containment and control measures of the related risks.

Strengthening of this competence is decisive and is a precondition for the epidemiological surveillance program. This shall occur through the qualification of the professionals involved, allowing recognition of the new pathogenic agents and diseases and fast intervention so as to define prompt and appropriate strategies. Moreover, there is still the need for implementation of improvements to hospital conditions and to the training of professionals dedicated to the assistance to suspected patients, and confirmed cases of pathogens of epidemiological relevance and risk of spreading, or cause of an emerging disease which becomes epidemiologically important or whose transmission mechanism is unknown.

As depicted at Figure 4, in 2006, the number of diseases whose etiological causes of agents of an unknown origin was almost four times as much as the number of diseases whose etiological agents belonged to risk group 3. Conversely, in 2007 (by April), the number of cases of these two categories had matched. The data, in the cases of diseases of unknown origin, demonstrate need for an effective health surveillance system, involving analysis of the outbreaks and field epidemiological investigation with the purpose of locating the transmission chain links. Frequently, in the unusual outbreaks, there is need for exhaustive investigation, as they involve agents, causal factors, unknown forms of transmission and dissemination and the number of persons afflicted may be high.

Certain epidemic events, which are featured as abnormal situations, are depicted to the community as potentially serious, and tend to trigger social pressures. Thus, they need to be adequately treated, which demands solutions from the authorities

Figure 4: Number of disease cases reported to CIEVS, in the country, from March 2006 to May 2007, caused by group risk 3 infectious agents and those by unknown agents.

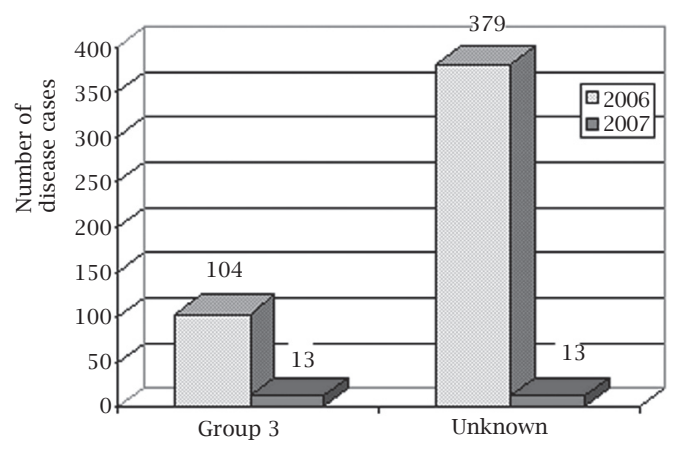
country, from March 2006 to May 2007, caused by group risk 3 infectious agents and unknown agents.

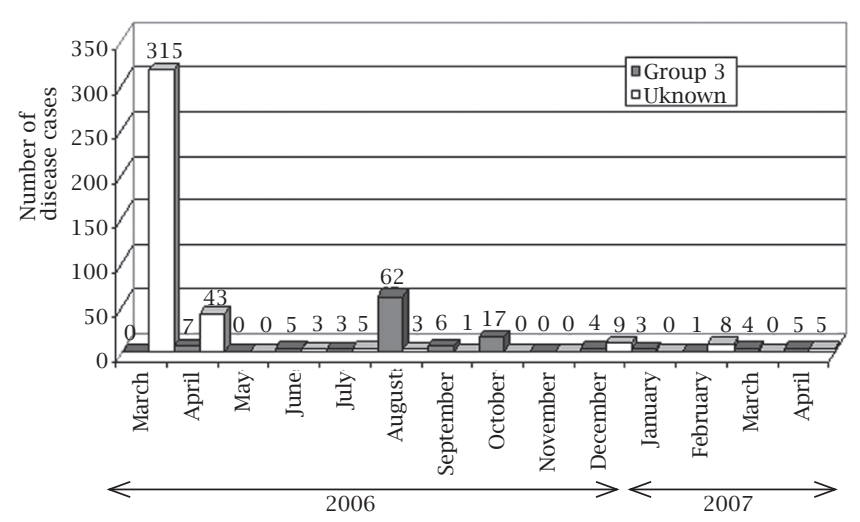

as expeditiously as possible, thereby conditioning the pace and conditions of the course of their investigation.

Prevention of new cases is priority in the ongoing outbreaks. The investigation should concentrate itself on the extent and size of the event and on the features of the population at risk, so as to outline and develop appropriate control measures. In case the epidemic is already close to its end, one should prevent the appearance of new cases. Here, investigation should aim, especially, at identifying the factors which have contributed to the occurrence of the event.

When the outbreak is of unknown cause, source and form of transmission, the disease is serious and the development of the investigation shall allow for its identification. Control actions can and should be taken, empirically, prior to its conclusion. However, there are certain situations in which control actions can only be implemented following exhaustive investigations. Seriousness of the event is a factor conditioning urgency in the course of investigation and in the implementation of control measures.

All professionals involved in the epidemiological investigation of an outbreak need to know the risks which they are exposed to. Soares ${ }^{16}$ points out that those professionals need to have an understanding of the epidemiological structure of the biological agents, including sources of infection and routes of transmission, as these factors comprise fundamental determinants in the prevention and reduction of contamination risk by environmental or biological sources during work performance.

An outbreak investigation involves a prior evaluation of the risks found in the work stages, from the planning and handling of infecting materials to the conditions for program performance, requiring training and discipline, as any failure can lead to contamination by direct or indirect contact with pathogenic material.

Figure 5 presents the number of cases occurring in each event reported, per month and year, of the illnesses whose etiological agents belong to risk group 3, and of

Figure 5: Number of disease cases reported to CIEVS, in the 
the diseases which have not had their etiological agent diagnosed yet (unknown).The high number of cases in the month of March was due to an unknown-etiology disease, albeit restricted to ophthalmologic symptoms, in the city of Araguatins (TO). This event, by its symptoms, demonstrated not having stemmed from a high pathogenic agent.

One should assign special importance to the cases reported in April, as they displayed symptoms of acute respiratory insufficiency, with blood spillage from the airways and cardiac-pulmonary syndrome (occurring in Natal (RN) and Alta Floresta (MT) respectively). These events depict symptoms which evidence airborne transmission, enhancing the risk.

There are factors related to infectious diseases in epidemic outbreaks which determine differentiated Biosafety procedures during epidemiological investigation. The one pertaining to the natural history of the disease, which covers the knowledge of disease evolution in the individual, stands out. Normally, one ascertains a period of anatomical or functional changes which highlight the subclinical or unapparent phase, which culminates with the beginning of the symptoms (incubation period). However, although diseases are unapparent during the incubation period, a number of pathological changes can be detected during this phase by means of laboratory methods, although most of the time diagnosis may only come up through symptoms. This fact is exacerbated by the instance that a considerable portion of the cases remains below the clinical horizon and, therefore, are not identified by their symptoms. Waldman ${ }^{17}$ points out still that "the higher the proportion of unapparent cases, the greater will be the difficulties to learn the infections processes, and to identify the main responsible agents for disease transmission within the community, as known cases represent only the tip of the iceberg". This shows that the possibility of a disease whose etiological agent is unknown and with a long period of incubation, transmitted by airborne agents is quite high. Thus, there is need for immediate implementation of control and prophylaxis methods in the region affected, such as isolating cases during the transmissibility period (when known); measures aiming at preventing or mitigating the disease, its complications and consequences; quarantine or isolation of the healthy population (human or animal) follow-up of the reported cases following the date of the last contact with an infected person. Compliance with the Biosafety principles is of paramount importance at all stages of epidemiological investigation, in the sense of preventing the spreading of pathogens; the capture, handling and safe transport of biological samples; assistance to the population affected and protection to the professional himself, so that the latter does not become a spreading source of the pathogenic agent.
Figure 6 presents the total cases of diseases reported caused by etiological agents from risk group 3 and unknown, per region, and per Brazilian state, in the period reviewed. The following was observed in data analysis:

Diseases with unknown etiological agent:

The North Region displays the highest number of these diseases (80.4\%) - the city of Araguatins (TO) presented the highest number of cases, albeit restricted to ophthalmological symptoms, with 306 cases of persons affected in March 2006, with no deaths. There was also an outbreak in this region, in the city of Novo Repartimento (PA), affecting 9 persons, with 6 deaths.

The Northeast Region concentrates, next, the highest number of these occurrences $(8.4 \%)$. It is important to point out the RN outbreak with 26 persons affected, in the city of Natal, in April 2006, with acute respiratory failure and bleeding in the airways, in which there were 6 deaths.

There were two outbreaks in the Southeast Region; one, in RJ (Campos dos Goytacazes), in December, affecting 5 persons with 4 deaths and another outbreak in MG (Frei Gaspar), in December, affecting 9 persons with 4 deaths. The total reported cases in this region placed it in the third position in the number of cases $(6.9 \%)$.

Despite the Central West Region not presenting a high percentage of reports $(4.0 \%)$, we should highlight the outbreak in the city of Alta Floresta (MT), in April, affecting 15 children in the 2-to-5-month-old age bracket, with $100 \%$ of deaths with cardiovascular syndrome symptoms.

Diseases caused by biological agents of risk group 3 biological agents:

The Central West Region presented the highest number $(43.5 \%)$ - MT reported, in the city of Campo Novo do Parecis, an outbreak of hantaviruses, affecting 39 persons with 5 deaths.

Figure 6: Number of cases reported to CIEVS, in Brazil, from March 2006 to May 2007, caused by diseases involving group risk 3 infectious agents and unknown, shown by State / Region.

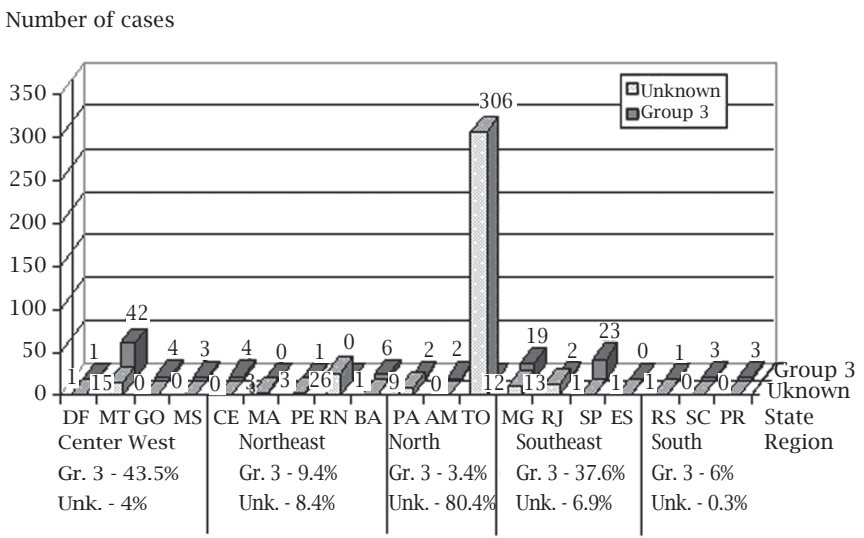


The Southeast region was the second region in number of reports $(37.6 \%)$ - the city of Campinas (SP), presented an outbreak of Spotted Fever, in August 2006, affecting 19 persons with 3 deaths, and the city of Caeté (MG), displayed another outbreak of Spotted Fever affecting 15 persons with one death.

By correlating the information on the number of reported diseases, per state, with the number of cases of persons affected by diseases involving risk group 3 etiological and unknown agents, one observes that, despite SP having presented the highest number of diseases reported in the period reviewed, it was not the state affected by diseases caused by etiological agents of higher epidemiological impact (risk group 3 or unknown). This means that the total number of events, even if it is significant, may not be of epidemiological relevance, regarding the spreading risk, transmissibility, and lethal potential, in the same way as a reduced number of events, but which have a significant quantitative incidence.

Figure 7 was built to show the country's geographic distribution of diseases caused by unknown etiological agents.

Figure 7: Distribution of cases reported to CIEVS, with unknown etiologic agents, according to the State, from March 2006 to May 2007.
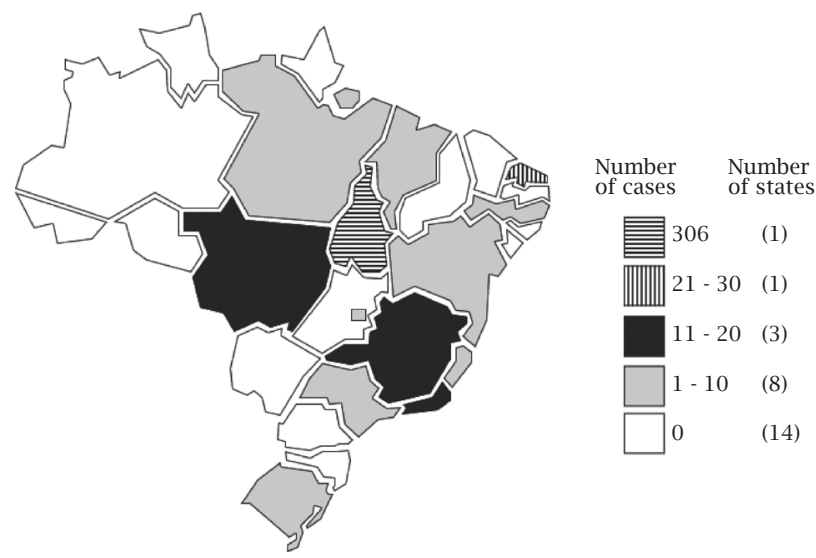

\section{Lethality}

The measurement of disease frequency and of deaths comprises a prerequisite for professionals developing routine surveillance activities and outbreak investigation. It is fundamental that this measurement is performed in the appropriate manner, so as to allow the featuring of risk of a specific disease to the population or estimate the magnitude of the health problem. ${ }^{17}$

Figure 8 data show the rate of lethality of reported diseases of unknown agents. The states of MT, MA, PE, ES, RS and DF stand out with $100 \%$ of lethality. However, it is important that $\mathrm{RN}$ and $\mathrm{DF}$ have only presented the report of one case whose outcome was death. The states of BA, TO and SP have presented cases of diseases of unknown etiology; however, these cases led to no deaths.

Out of a total of 392 cases of diseases whose etiological agents were unknown, 53 deaths were ascertained, that is, $13.5 \%$ of lethality, which is representative and which expresses the need for a deeper evaluation of their causes.

Figure 8 shows a spatial distribution of the lethality rate of diseases, thereby demonstrating that there has been no concentration in any region.

Figure 8: Distribution of lethality rates and fatal cases, reported to CIEVS, with unknown etiologic agents, according to the State, from March 2006 to May 2007.

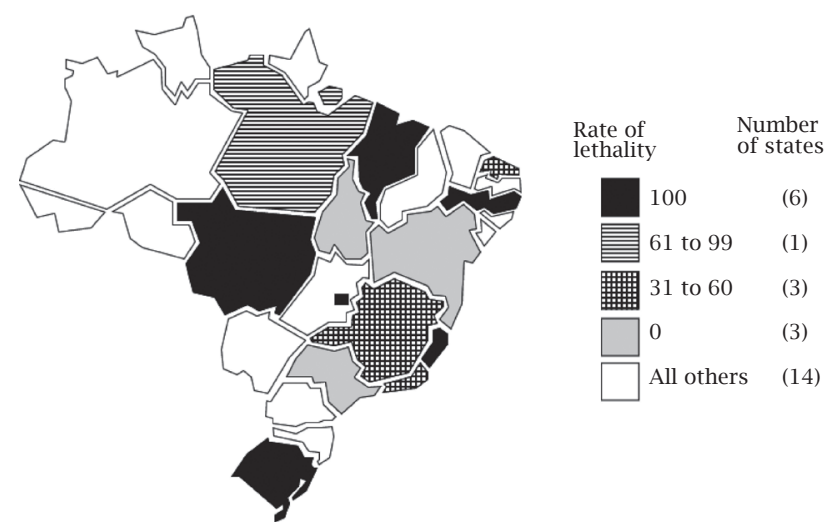

\section{CONCLUSION}

The context of the issues discussed in this study clearly shows the need for awareness in Biosafety in the health surveillance practices, especially in epidemiological investigations in Brazil.

The emergence and re-emergence of infectious diseases are phenomena which represent a constant and powerful force of nature, with the important participation of human behavior and its relationship with the environment. The occurrence of these diseases challenges the epidemiological investigations and public health as a whole. One of these challenges refers to Biosecurity and to Biosafety.

The hazards facing risk agents, most of the times of an unknown nature, requires that multidisciplinary teams be qualified to identify and appraise risks, so that prevention and control measures apply to the elimination or minimization of these risks, either for the professionals or for the environment.

At times, the investigation of an outbreak which presents itself initially as routine, may lead to the characterization of a disease hitherto unknown, as well as of its agents, sources and/or mode of transmission and control measures, representing high risk to the professionals involved 
in the investigation and which could only be handled under maximum containment conditions. We mention, as an example, the characterization of the legionnaire's disease and of the acquired immunodeficiency syndrome, when an outbreak of pneumonia was being investigated, and which afflicted a group of individuals belonging to a North American Civil organization named "American Legion", and another outbreak of pneumonia by Pneumocystis carinii among young homosexuals. In Brazil, the Brazilian purpuric fever, is another example, individualized as a new disease framework, during the investigation of a fulminans purpuric outbreak, which occurred in the municipality of Promissão (SP), affecting children below 10 years of age, with high rate of lethality, not associated to infection by Neisseria meningitidis and by $H$. influenzae type B, which had, until then evidently determined this syndrome.

It is necessary, in confronting outbreaks, to dispose of, or feature changes to disease, agent source or mode of transmission behavior. This is what happened, in the 1980's, with the poliovirus type 3 epidemics, which affected the Brazilian Northeast. The possibility of the poliovirus to comprise a mutant virus was investigated, as the population involved in the episode was supposedly immunized. There is still the risk that new, highlylethal etiological agents, multi-drug-resistant agents, the use of genetic engineering techniques in the mutation of pathogen genes, causes changes in features (stability, virulence, spreading property etc.), leading to the possibility of their being used as biological weapons, which has been mobilizing the security forces of developed countries. ${ }^{18}$ However, even more important than this danger is the quite real possibility of global traffic of viruses in a few hours, from a continent, by means of air trips.

Taking into account the scientific and technological resources, in which Biosafety originally built and consolidated its attraction the BSL3 laboratories are used for the study of biological agents which are potentially lethal, transmissible by aerosols, and which require specific secondary barriers, for greater containment, such as a specialized air system. The BSL4 laboratories are normally used for the study of lethal biological agents, whose treatment or immunoprophylaxis does not exist. They incorporate the specificity of containment of the BSL3 added to additional containment barriers such as positive pressure clothing with life support systems. Generally, Biosafety precautions are determined by the risk of biological agents handled and the type of assay to be developed. However, risk assessment has to be performed prior to activity performance, in which aspects pertaining to the workers themselves are taken into consideration. Additional security protection may be necessary, at any level of Biosafety, from 1 to 4, depending on the specific needs of the assay to be developed.

The National Institutes of Health reports the existence of 277 BSL3 laboratories in the USA, until 2005. ${ }^{19}$ However, we have been facing a growth in the number of these types of laboratories throughout the world. A number of BSL3 laboratories have been built, and went into operation in
2006: in India (16 new laboratories), Thailand (5), Indonesia (2), Bangladesh (1), and Myanmar (1). ${ }^{20}$ The increase in the number of BSL3, as well as the BSL4 laboratories is due, especially, to the increment of research and adequacy requirements of these research works to the Biosafety and Biosecurity rules.

Brazil's great biodiversity variety includes etiological disease agents, able to cause damage to public health and to the environment and which could be used for the development of biological weapons. The Brazilian Sabiá virus is an example.

To face this new situation, so that one may ensure a minimum of self-support to the National Health System, it is indispensable that the following instruments to public health practices are incorporated:

- Surveillance in public health, in the epidemiological intelligence sense, as an induction instrument for research, for strategies of disease control and incorporation of the knowledge produced;

- Epidemiological and laboratory research;

- Health services organized in a way to incorporate regularly, in an agile manner, new knowledge and technologies indispensable to the continuous elaboration, evaluation and reformulation of strategies for disease control, considering Biosafety among them on a priority basis.

The discussion developed evidences the complexity which is being drawn up in the Biosafety field as this field works and establishes interlocutions with the field of application of technologies, biological agent manipulation and the important and growing demand for risk control.

It is important to mention that the process of development of vaccines and pharmaceutical products starts with the understanding of the biology of the biological agents involved and the pathogenesis of the disease they cause. Many of these studies should be performed at BSL3 or 4, and at containment laboratories, for the growth process of the organism and extraction of its nucleic acid and proteins. The transmission, disease progression, and effectiveness of drugs and vaccines are performed with in vivo assays of high complexity and containment.

Research investments must assign priority to areas such as: the development of laboratory techniques of diagnostic kits; knowledge of the magnitude and dispersion of diseases and their risk factors; elucidation of the transmission chain links; explanation for the resistance phenomena to medications or to insecticides, development and evaluation of new vaccines and drugs. The existence of laboratory facilities which bear appropriate containment requirements (including BSL4), well structured and trained professional teams for diagnosis, well structured and trained, and a well defined, easily accessible laboratory hierarchy are necessary for such purpose. 


\section{REFERENCES}

1. Cardoso TAO, Navarro MBMA. Emerging and reemerging diseases in Brazil: data of a recent history of risks and uncertainties. Braz J Infect Dis 2007; 11:430-4.

2. Navarro MBMA, Cardoso TAO. Biossegurança e Ambiente: Complexidade e Instrumentalização. Gaia Scientia 2007; 1:107-14.

3. Luna EJA. The emergence of emerging diseases and emerging and re-emerging infectious diseases in Brazil. Rev Bras Epidemiol 2002; 5:229-43.

4. Possas CA. Social ecosystem health: confronting the complexity and emergence of infectious diseases. Cad Saúde Pública 2001; 17:31-41.

5. Marques MB. Emerging infectious diseases in the realm of complexity: implications for scientific and technological policies. Cad Saúde Pública 1995; 11:361-71.

6. Schmidt RAC. A questão ambiental na promoção da saúde: uma oportunidade de ação multiprofissional sobre doenças emergentes. Physis 2007; 17:373-92.

7. Hammann EM, Laguardia J. Reflexões sobre a vigilância epidemiológica: mais além da notificação compulsória. Inf Epidemiol SUS 2000; 9:211-9.

8. Franklin R, Cockerill III, Thomas FS. Response of the clinical microbiology laboratory to emerging (New) and re-emerging Infectious Diseases. J Clin Microbiol 2004; 42:2359-65.

9. Barata RCB. The challenge of emergent disease and the return to descriptive epidemiology. Rev Saúde Pública 1997; 31:531-7.

10. Carmo EH, Barreto ML. Mudanças nos padrões de morbimortalidade da população brasileira: os desafios para um novo século. Epidemiologia e Serviços de Saúde 2003; 12:63-75.

11. Rocha SS, Guerra C. A Biossegurança no Projeto de Qualidade do LACEN. LACEN-INLACEN 2000 Jan 4; p. 1.
12. Cardoso TAO. Análise da construção da competência do Brasil em direção ao Laboratório de Contenção Máxima: realidades e perspectivas. Rio de Janeiro, 208p. Tese de (Doutorado) Escola Nacional de Saúde Pública, Fundação Oswaldo Cruz, 2008.

13. Brasil. Ministério da Saúde. Portaria $n^{\circ} 5$. Relação Nacional de Doenças de Notificação Compulsória; 2006.

14. Centers for Disease Control and Prevention. Principles of epidemiology. An introduction to applied epidemiology and biostatistics. Atlanta: Centers for Disease Control and Prevention; 2006.

15. Brasil. Ministério da Saúde. Comissão de Biossegurança em Saúde. Classificação dos Agentes Biológicos. Brasília, DF: Ministério da Saúde; 2006.

16. Soares BEC. Biossegurança no Trabalho de Campo. In: Oda LM, Ávila SM (eds). Biossegurança em Laboratório de Saúde Pública. Brasília, DF: Ministério da Saúde; 1998.

17. Waldman EA. Vigilância em Saúde Pública. São Paulo, SP: Faculdade de Saúde Pública da USP; 1998.

18. Crane JAIA. Emerging Challenges in Containment: development of a biodefense infrastructure. In: Richmond JY (ed). Anthology of Biosafety VIII. Mundelein, IL: ABSA; 2005.

19. National Institutes of Health. Final Report: 2005. Survey for determining the location, capacity, and status of existing and operating BSL3 laboratory facilities within the United States. [Online] http://www3.niaid.nih.gov/biodefense/PDF/BSL3_survey.pdf 2008.

20. Gronvall GK, Fitzgerald J, Chamberlain A, Inglesby A, O’Toole T. High-Containment Biodefense Research Laboratories: meeting report and center recommendations. Biosecurity and Bioterrorism: biodefense strategy, practice, and science 2007; 5:75-85. 